\title{
Mechanism Design for Energy Efficiency in Wireless Networks
}

\author{
Anil Kumar Chorppath, Tansu Alpcan, \\ Technical University of Berlin \\ Deutsche Telekom Laboratories \\ Email: anil.chorppath@sec.t-labs.tu-berlin.de, \\ alpcan@sec.t-labs.tu-berlin.de
}

\begin{abstract}
Network mechanism design aims to achieve system level goals such as efficiency or social welfare maximization through resource allocation mechanisms on networks, where individual users are selfish and independent decision-makers. This paper focuses on mechanisms for energy efficiency in uplink of multicarrier wireless systems and rate control in wireless networks. The problem is modeled as strategic (noncooperative) game with a resource pricing scheme, where the prices are imposed by a mechanism designer. The users decide on their actions according to both own preferences and given prices. The rules and incentives of the mechanism are designed in such a way that the system objective which is a combination of social welfare and energy efficiency is maximized. A weighting parameter in the objective function allows to smoothly vary the emphasis from social welfare to energy efficiency according to the preference of the designer. The users are assumed initially to be concerned only about their throughput. However, the designer modifies their incentives using pricing so that they become more energy-aware. A distributed optimization framework is developed accordingly in which users have general concave utilities (in terms of throughput) that are unknown to the designer and the energy-efficiency objective is expressed as a convex function of user power levels. An iterative pricing mechanism is obtained as a result, and illustrated through simulations.
\end{abstract}

\section{Introduction}

In mechanism design, a designer imposes rules on an underlying strategic (noncooperative) game in

This work has been supported by Deutsche Telekom Laboratories. order to achieve certain desirable objectives such as energy efficiency or social welfare maximization for the users, who are the players of the underlying game. Hence, mechanism design can be viewed as reverse engineering of strategic games with the goal of achieving certain system level objectives. There has been widespread interest in using mechanism design for modeling, analyzing and solving problems in network resource allocation that are decentralized in nature [1][4]. However, majority of these works focus on social welfare maximization.

Recently, researchers have become more aware of environmental concerns and the need for energy efficient protocols in all types networks. There has been significant amount of work in the context of Adhoc [5], [6] and sensor networks [7] to obtain energy efficient protocols. In [8], a game theoretic model is proposed for energy efficient power control by defining utility of users as the ratio of throughput (goodput) and power (with unit bit/J) for multi-carrier CDMA wireless systems. A repeated game model and the cooperation induced due to repeated interaction is analyzed in [9]. We note that most of the previous works on game models for energy efficiency modify user utilities by incorporating a power term into the user utility function [8]-[10], assuming that users care for their energy usage in uplink transmission. In contrast, we consider here the alternative scenario and assume that the users care only for getting maximum throughput, but an external mechanism designer imposes prices to the users such that their energy consumption is decreased to improve overall energy efficiency of the system. Thus, the designer encourages users to be more energy conscious. In addition, we also consider user utility dependence on higher layer parameters.

We model energy efficiency objective by subtracting a general convex function of the power levels of users from the social welfare (sum of utilities of all 
users). This additional term is multiplied by a tuning parameter which allows smoothly varying the emphasis from the social welfare to the system energy efficiency.

As a way of improving energy efficiency, we make use of pricing mechanisms (see e.g. [11] and references therein) for single cell uplink transmission of a multi-carrier wireless system. The users decide on their individual power level and get a per unit price depending on their own actions as well as those of all other users. The underlying game of the mechanism converges to a Nash equilibrium iteratively in a way that the users respond to the prices set by the designer. Here, the users are considered to be price taking since they are not minimizing their cost anticipating the price which is justified by their lack of global information. The pricing mechanism framework in this paper builds upon the results in [12].

The rest of the paper is organized as follows. The next section presents the underlying model and assumptions mechanism design framework. In Section 3, pricing mechanisms for energy efficient multi-carrier wireless systems are proposed. In a similar way energy efficient pricing mechanisms are obtained for rate control in wireless networks in Section 4. Simulation setting is discussed and results are given in Section 5. The paper concludes with a summary and description of ongoing work in Section 6.

\section{Model}

At the center of the game and mechanism design model is the designer $\mathcal{D}$ who influences $N$ players, denoted by the set $\mathcal{A}$, and participating in a noncooperative game. Let us define an $M$-player strategic game, $\mathcal{G}$, where each player $i \in \mathcal{A}$ has a respective decision variable $x_{i}$ such that

$$
x=\left[x_{1}, \ldots, x_{M}\right] \in \mathcal{X} \subset \mathbb{R}^{M \times N},
$$

where $\mathcal{X}$ is the decision space of all players. Let

$x_{-i}=\left[x_{1}, \ldots, x_{i-1}, x_{i+1}, \ldots x_{M}\right] \in \mathcal{X}_{-i} \subset \mathbb{R}^{(M-1) \times N}$,

be the profile of decision variable of players other than $i^{t h}$ player and $\mathcal{X}_{-i}$ is the respective decision space. This paper assumes vector decision variables which represent the power level users take over each carrier and a compact and convex decision space. Due to the inherent coupling between the players due to the interference in wireless systems, the decisions of players directly affect each other's performance as well as the aggregate allocation of limited bandwidth. Each user decides on the power level over the $N$ carriers. Therefore,

$$
x_{i}=\left[x_{i}^{(1)}, \ldots, x_{i}^{(N)}\right] \text {, }
$$

where $x_{i}^{(n)}=h_{i}^{(n)} p_{i}^{(n)}$ denote the received power level over carrier $n$ as a product of uplink transmission power $p_{i}^{(n)}$ and channel loss $0<h_{i}^{(n)}<1$ of player $i$. If linear interference is assumed, then the signal-tointerference-noise ratio (SINR) of the received signal on channel $n$ is

$$
\gamma_{i}^{(n)}=\frac{x_{i}^{(n)}}{I_{i}^{n}},
$$

where $I_{i}^{n}=\sum_{j \neq i} x_{j}^{(n)}+\sigma$ is the interference experienced by user $i$ and $\sigma$ is the background noise power.

The users have utility functions

$$
U_{i}(x):=\sum_{n} U_{i}\left(\gamma_{i}^{(n)}(x)\right): \mathcal{X} \rightarrow \mathbb{R}, \quad \forall i \in \mathcal{A} .
$$

In this paper, the utility functions are assumed to be monotonically increasing, differentiable and concave function of player SINR, and assumed to be unknown to the designer.

The designer $\mathcal{D}$ devises a mechanism $M$, which can be represented by the mapping $M: \mathcal{X} \rightarrow \mathbb{R}^{N}$, implemented by introducing incentives in the form of rules and prices to players. The latter can be formulated by adding it as a cost term such that the player $i$ has the cost function

$$
J_{i}(x)=c_{i}(x)-U_{i}(x) .
$$

where $c_{i}(x)$ is the total payment imposed to the player by the designer. In the case of pricing mechanisms,

$$
c_{i}(x)=x_{i} P_{i}(x),
$$

where $P_{i}(x)$ is the per unit price.

The player objective is to obtain optimum power levels as a best response to the action of other players by solving the following individual optimization problem in the game

$$
\min _{x_{i}} J_{i}\left(x_{i}, x_{-i}\right), \forall i
$$

under the given constraints of the strategic game, and rules and prices imposed by the designer.

Similar to player preferences, the designer objective, can be formulated using a smooth objective function $V$ for the designer:

$$
V\left(x, U_{i}(x), c_{i}(x)\right): \mathcal{X} \rightarrow \mathbb{R},
$$

where $c_{i}(x)$ and $U_{i}(x), i=1, \ldots, N$ are userspecific pricing terms and player utilities, respectively. Conventionally, achieving the global objective (e.g. maximization of aggregate user utilities) requires interference from the designer to the underlying game in terms of designing payments as a cost of control. The minimization of interference from the designer 
to the underlying game is modeled by adding a cost of control term to the global objective. The resulting global objective formulation is actually well-known in optimal control theory and widely used in a variety of contexts [14].

We consider here the global objective as the social welfare minus a convex energy efficiency term, tuned appropriately using the parameter $\phi$. This unconventional objective is given as

$$
V(x)=\sum_{i} U_{i}(x)-\phi R(x) .
$$

where $0 \leq \phi \leq 1$ is the tuning parameter and $R(x)$ is any convex function on $x$ that captures the cost on energy usage. It is similar to cost of control in other settings. Due to the convexity of the additional term, we can see that the users sacrifice much on their net utility if they transmit with higher power. A specific example function is, $R(x)=\sum_{i} R_{i}\left(x_{i}\right)$, where $R_{i}\left(x_{i}\right)$ can be any convex function of $x_{i}$. We refer to efficient mechanisms here as those which maximize designer objective, i.e. they solve the problem $\max _{x} V\left(x, U_{i}(x), c_{i}(x)\right)$. Hence, the global objective of the designer is to obtain efficient results that are achieved indirectly by setting prices.

\section{Pricing Mechanisms for Energy Effi- cient Wireless Systems}

In pricing mechanisms, the designer charges the players for their resource usage and players take actions in response to that. Pricing mechanisms are applicable to many networked systems where an explicit allocation of resources brings a prohibitively expensive overhead or simply not feasible, e.g. due to participating players being selfish or located in a distributed manner. The optimality conditions obtained from (4) using the first derivative are given by,

$$
\begin{gathered}
\frac{d U_{i}}{d x_{i}^{n}}-P_{i}^{n}=0, \forall n, i, \\
\frac{d U_{i}}{d \gamma_{i}^{(n)}}=P_{i}^{(n)} I_{i}^{(n)}, \forall i \in \mathcal{A}, n .
\end{gathered}
$$

The global objective of designer with individual user power constraint is to solve the optimization,

$$
\max _{x} \sum_{i} U_{i}(x)-\phi \sum_{i} R_{i}\left(x_{i}\right)
$$

such that

$$
\sum_{n} \frac{x_{i}^{(n)}}{h_{i}^{(n)}} \leq P_{\max },
$$

where $P_{\max }$ is the maximum allowable power to indi- vidual users. The constraint on the maximum allowable power level will be set by the regulating authority due to the limit on total interference. The designer, apart from this requirement, is concerned about the total energy consumption in the cell. The nature of the designer, i.e, the extend to which she cares about energy efficiency, is captured in the parameter $\phi$. Thus, the Lagrangian function of the designer problem can be written as:

$$
\begin{aligned}
L & =U_{i}(x)+\sum_{j \neq i} U_{j}(x)-\phi \sum_{i} R_{i}\left(x_{i}\right) \\
& -\sum_{i} \lambda_{i}\left(\sum_{n} \frac{x_{i}^{(n)}}{h_{i}^{(n)}}-P_{\max }\right),
\end{aligned}
$$

where $\lambda_{i}$ 's are the Lagrangian multipliers.

This problem can be solved by convexification by the transformation $x_{i}=e^{s_{i}}$ as described in [13]. The corresponding Karush-Kuhn-Tucker (KKT) conditions are:

$$
\begin{gathered}
\frac{d U_{i}}{d x_{i}^{(n)}}+\sum_{j \neq i} \frac{d U_{j}(x)}{d x_{i}^{(n)}}-\phi \frac{d R_{i}}{d x_{i}^{(n)}}-\frac{\lambda_{i}}{h_{i}^{(n)}}=0, \forall i, n, \\
\lambda_{i}\left(\sum_{n} \frac{x_{i}^{(n)}}{h_{i}^{(n)}}-P_{\max }\right)=0 .
\end{gathered}
$$

We next align the solution of both designer and user problems, and obtain the price and action vectors that solve all of them concurrently. By combining the above KKT conditions and the conditions of user best response from (6), we obtain the prices as:

$$
P_{i}^{(n)}=\phi \frac{d R_{i}}{d x_{i}^{(n)}}-\sum_{j \neq i} \frac{d U_{j}(x)}{d x_{i}^{n}}+\frac{\lambda_{i}}{h_{i}^{(n)}} \forall i, n
$$

Consider for demonstration purpose the energy cost term as

$$
R_{i}\left(x_{i}\right)=\frac{\sum_{n}\left(p_{i}^{(n)}\right)^{2}}{2}=\frac{1}{2} \sum_{n} \frac{\left(x_{i}^{(n)}\right)^{2}}{\left(h_{i}^{(n)}\right)^{2}} .
$$

Notice that due to the weighting by channel coefficients, good channels are encouraged in this case while bad ones are discouraged.

In this special case, the prices from (9) can be written using (7) and definition of $\gamma_{j}^{n}$ as,

$$
P_{i}^{(n)}=\sum_{j \neq i} \gamma_{j}^{n} P_{j}^{n}+\phi \frac{x_{i}^{(n)}}{\left(h_{i}^{(n)}\right)^{2}}+\frac{\lambda_{i}}{h_{i}^{(n)}}, \forall i, n .
$$

The above set of equations can also be written in matrix form as,

$$
A^{(n)} \cdot P^{(n)}=B^{(n)} \cdot L^{(n)}, \forall n,
$$


where the matrices $A^{(n)}$ and $B^{(n)}$ are defined as

$$
\begin{aligned}
A^{(n)}: & =\left(\begin{array}{cccc}
1 & -\gamma_{2}^{(n)} & \cdots & -\gamma_{N}^{(n)} \\
-\gamma_{1}^{(n)} & 1 & \cdots & -\gamma_{N}^{(n)} \\
\vdots & & \ddots & \vdots \\
-\gamma_{1}^{(n)} & -\gamma_{2}^{(n)} & \cdots & 1
\end{array}\right), \quad(11) \\
B^{(n)}:= & \left(\begin{array}{cccccc}
\frac{1}{h_{1}^{(n)}} & \cdots & 0 & \frac{\phi}{\left(h_{1}^{(n)}\right)^{2}} & \cdots & 0 \\
0 & \cdots & 0 & 0 & \cdots & 0 \\
\vdots & & \ddots & \vdots & \vdots & \\
0 & \cdots & \frac{1}{h_{N}^{(n)}} & 0 & \cdots & \frac{\phi}{\left(h_{N}^{(n)}\right)^{2}}
\end{array}\right)
\end{aligned}
$$

and $L^{(n)}=\left[\lambda_{1}, \ldots, \lambda_{N}, x_{1}^{(n)}, \ldots, x_{N}^{(n)}\right]^{T}$.

Consider the example where users have their utility as logarithmic of the received SINR, i.e.,

$$
U_{i}(x)=\alpha_{i} \sum_{n} \log \left(\gamma_{i}^{(n)}(x)\right)
$$

where $\alpha$ is user preference vector unknown to the base station. Then,

$$
\frac{d U_{i}}{d x_{i}^{n}}=\frac{\alpha_{i}}{x_{i}^{n}}=P_{i}^{(n)}
$$

and

$$
\frac{d U_{j}}{d x_{i}^{n}}=\frac{-\alpha_{j}}{\left(\sum_{k \neq j} x_{j}^{(n)}+\sigma\right)^{2}} .
$$

This example is used for the simulations as given in Section 5.

\subsection{Distributed Iterative Energy Efficient Pricing Algorithm}

We now define the pricing mechanism $\mathcal{M}^{e}$, where the prices and user power levels (actions) for each carrier are obtained using iterative methods, by adopting a greedy update scheme for both user power levels and prices:

$$
\begin{gathered}
P(k+1)=(A)^{(n)^{-1}} B^{(n)} \cdot L(k), \forall n \\
p_{i}^{(n)}(k+1)=p_{i}^{(n)}(k)-\frac{\kappa_{i}}{h_{i}^{(n)}} \frac{\partial J_{i}}{\partial p_{i}^{(n)}} \forall i \in \mathcal{A}, \quad \text { (14) } \\
\lambda_{i}(k+1)=\left\{\begin{array}{c}
\lambda_{i}(k)+\kappa_{D}\left(\sum_{n} \frac{x_{i}^{(n)}(k)}{h_{i}^{(n)}}-P_{\max }\right), \\
\text { if } \sum_{n} \frac{x_{i}^{(n)}(k)}{h_{i}^{(n)}} \geq P_{\max } \\
0, \text { otherwise }
\end{array}\right.
\end{gathered}
$$

Since the designer optimization problem can be convexified and thus admits a unique solution, we can find unique Lagrange multipliers ( $\lambda$ 's) which align it to the user convex optimization problems.

The implementation of mechanism $\mathcal{M}^{e}$ requires minimum information overhead. The designer only needs to observe the received power level vector $x$ and the individual SIRs, $\gamma$, of players both of which are already available. The player $i$, in return only needs to know the current price $P_{i}$ to be able to compute the (gradient) best response. Finally, the computation of actual uplink power levels $p$ can be carried from $x$ using the measured channel gains.

The algorithm which also shows the information flow for the iterative method is given below in Algorithm 1.

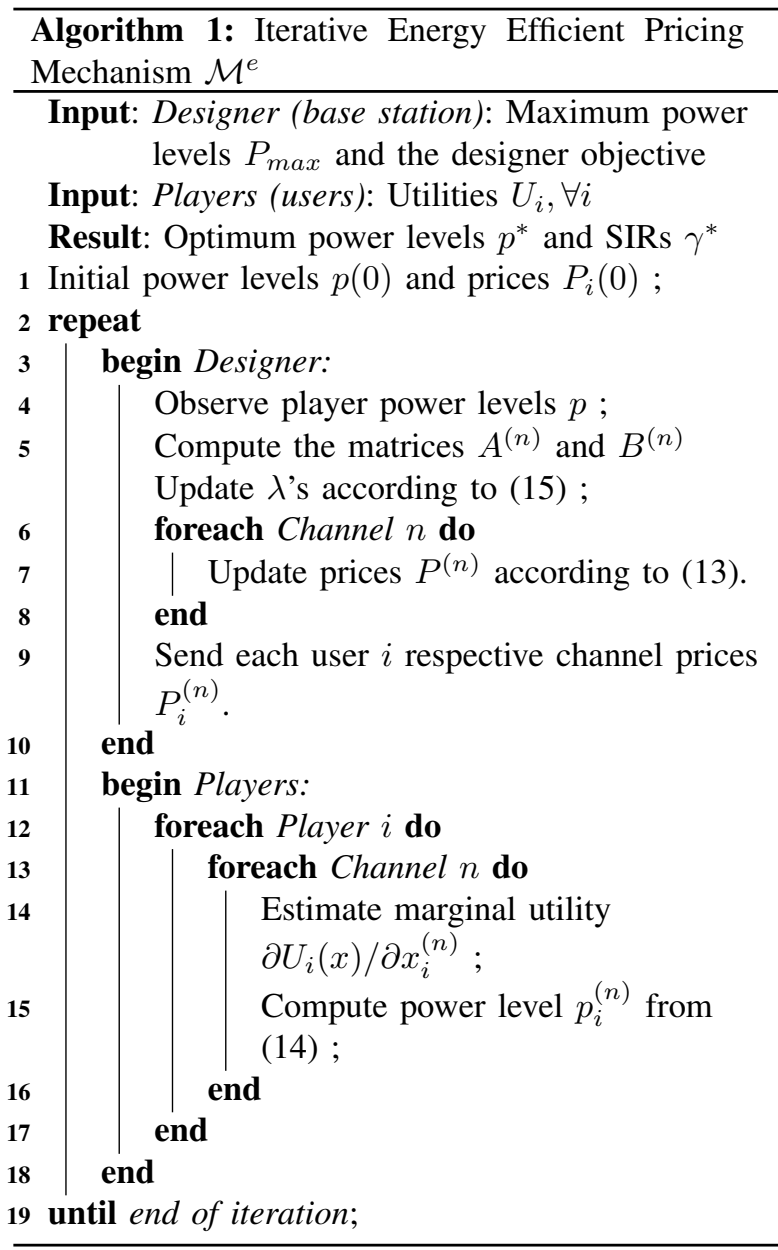

\section{Pricing Mechanisms for Energy Effi- ciency in Rate Control}

We consider here the rate control in wireless networks [11] in which the utility function for each user 
is a separable function of their individual rates. This is a case of additive resource sharing, in which share the fixed resource $C$. The users are assumed to have monotonically increasing, differentiable and concave utility function of their rate $U_{i}\left(x_{i}\right)$, where $x_{i}$ is the individual rate.

The designer objective is to solve the optimization, $\max _{x} \sum_{i} U_{i}\left(x_{i}\right)-\phi \sum_{i} R_{i}\left(x_{i}\right)$ such that $\sum_{i=1}^{N} x_{i}=C$.

Then, the associated Lagrangian function and KarushKuhn-Tucker conditions are given as

$$
L(x)=\sum_{i} U_{i}\left(x_{i}\right)-\phi R_{i}\left(x_{i}\right)+\lambda\left(C-\sum_{i} x_{i}\right)
$$

and

$$
\lambda\left(C-\sum_{i} x_{i}\right)=0 .
$$

The optimality conditions assuming boundary solution are

$$
U_{i}^{\prime}\left(x_{i}\right)-\phi R_{i}^{\prime}\left(x_{i}\right)=\lambda \forall i
$$

and

$$
\sum_{i} x_{i}=C
$$

The optimal $\lambda^{*}$ and rate levels $x^{*}$ can be obtained by having an efficient mechanism $M_{r}$, which can be implemented in an iterative way similar to one in previous section.

\section{Simulations}

In this section, we conduct numerical simulations which illustrate the convergence of the power levels and Lagrange multiplier in the proposed energy efficient pricing mechanism. The users are assumed to have utility function as $U_{i}(x)=\alpha_{i} \sum_{n} \log \left(\gamma_{i}^{(n)}\right)$ and value of tuning parameter $\phi=0.8$. The users update their power levels according to (14) at each time step $k \geq 1$ with a step size of $\kappa=0.02$. The designer, on the other hand, updates the Lagrangian multipliers $\lambda$ 's and price vector $P$ based on (13) and (15), where $P_{\max }=0.9$ and $\kappa_{D}=0.02$. The background noise parameter is $\sigma=0.5$. The convergence of the mechanism $\mathcal{M}^{e}$ is depicted in Figures 1 and 2.

We could observe from the Figure 1 that the user power levels converge within an $\epsilon$ limit due to the approximation in the Lagrange multipliers update. Also, only the Lagrange multiplier corresponding to the user, whose power level hit the boundary, has nonzero value, as expected.

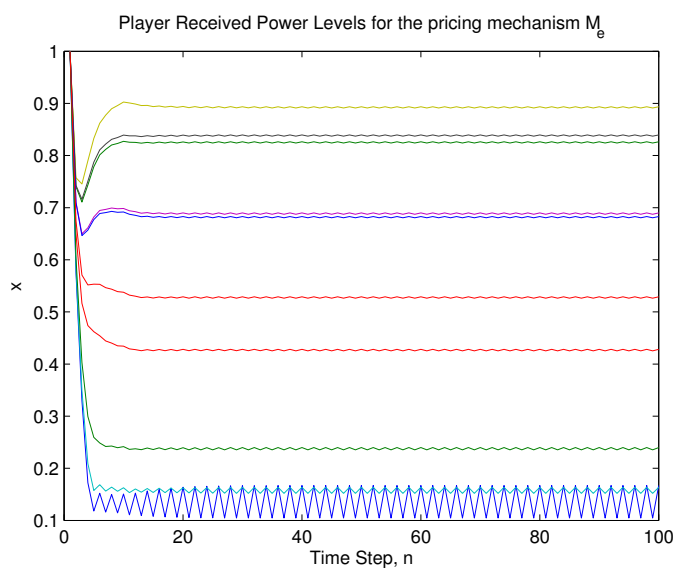

Figure 1. The evolution of user power levels $x$ in pricing mechanism $\mathcal{M}^{e}$ for a single carrier.

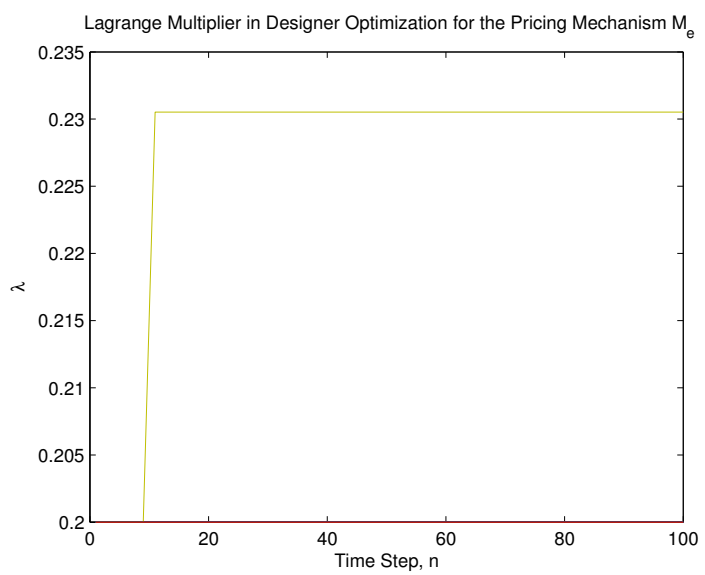

Figure 2. The evolution of Lagrange multiplier $\lambda$ in pricing mechanism $\mathcal{M}^{e}$ for a single carrier.

\section{Conclusion}

We have developed energy-efficient network mechanisms for wireless networks where the individual users are selfish and independent decision makers. For this, we have used an unconventional designer objective consisting of an aggregate utility minus a convex function of the user actions representing cost of energy usage. In our framework, the users need not report their utility function to the designer, which avoids high communication burden between the designer and the users. First, we proposed energy-efficient pricing mechanisms for power control in interference coupled multi-carrier wireless systems. An iterative algorithm is proposed to implement the pricing scheme developed. The results are extended for rate control in wireless networks in which users have separable utility 
functions. The numerical studies show the convergence of the power levels and Lagrange multipliers for the pricing algorithm proposed.

\section{References}

[1] F. P. Kelly and A. K. Maulloo and D. Tan, "Rate control in communication networks: shadow prices, proportional fairness and stability," Journal of the Operational Research Society, 1998, volume 49, pp. 237-252.

[2] J. Huang and R. Berry and M. Honig, "Auction-based Spectrum Sharing", ACM Mobile Networks and Applications Journal, pp.405-418, 2006.

[3] A. K. Chorppath, S. Bhashyam, and R. Sundaresan, "A convex optimization framework for almost budget balanced allocation of a divisible good, " IEEE Transactions on Automation Science and Engineering, January, 2011.

[4] S. Yang and B. Hajek, "VCG-Kelly Mechanisms for Allocation of Divisible Goods: Adapting VCG Mechanisms to One-Dimensional Signals", IEEE JSAC, August 2010.

[5] W. Feng and J.M.H. Elmirghani," Green ICT: Energy Efficiency in a Motorway Model," In Third International Conference on Next Generation Mobile Applications, Services and Technologies, 2009. NGMAST '09, pp. 389 - 394, September 2009.

[6] P. Bergamo and A. Giovanardi and R. Giovanardi and A. Travasoni and D. Maniezzo and G. Mazzini and M. Zorzi, "Distributed Power Control for Energy Efficient Routing in Ad Hoc Networks," Wireless Networks, vol. 10, pp. 29-42, 2004.

[7] R. K. Min, “ Energy and quality scalable wireless communication," Thesis (Ph. D.)-Massachusetts Institute of Technology, Dept. of Electrical Engineering and Computer Science, 2003.

[8] F. Meshkati, M. Chiang, H.V. Poor and S.C. Schwartz, "A game-theoretic approach to energy-efficient power control in multicarrier CDMA systems," IEEE Journal on Selected Areas in Communications, vol.24, no.6, pp.1115-1129, June 2006.

[9] M. Le Treust, S. Lasaulce and M. Debbah, "Implicit cooperation in distributed energy-efficient networks," In 4th International Symposium on Communications, Control and Signal Processing (ISCCSP), 2010 pp. 16, March 2010.

[10] D. J. Goodman and N. B. Mandayam, "Power control for wireless data,'IEEE Person. Comm., vol. 7, pp. 4854, 2000.

[11] R. Srikant, "The Mathematics of Internet Congestion Control", Systems \& Control: Foundations \& Applications, 2004.
[12] T. Alpcan and L. Pavel, "Nash Equilibrium Design and Optimization", Proc. of Intl. Conf. on Game Theory for Networks (GameNets 2009),Istanbul, Turkey, May, 2009.

[13] H. Boche and M. Schubert, "A Superlinearly and Globally Convergent Algorithm for Power Control and Resource Allocation With General Interference Functions", IEEE/ACM Transactions on Networking, pp.383-395, 2008, .

[14] D. E. Kirk, "Optimal Control Theory: An Introduction," Dover Publications, 2004. 